delayed to allow stabilisation of general condition. It should be stressed that whatever the timing, adequate debridement of devitalised tissue, tension-free approximation and use of nonabsorbable material are principles paramount to the success of the repair.

1 Clain A. Traumatic hernia. Br $\mathcal{F}$ Surg 1964;51:549-50. 2 Atiemo EA, Goswami G. Traumatic ventral hernia. Trauma 1974;14:181-2.

3 Dajee $H$, Nicholson DM. Traumatic abdominal hernia. Trauma 1979;19:710-11.

Trauma 1979;19:710-11.
Payne DD, Resnicoff SA, States JD, Williams JS. Seat bel abdominal wall muscular avulsion. $\mathcal{f}$ Trauma 1973;13:262-7.

\section{Final diagnosis}

Post-traumatic abdominal wall hernia.

Keywords: hernia; abdominal trauma

5 Malangoni $M$, Condon RE. Traumatic abdominal wall hernia. F Trauma 1983;23:356-7.

6 Dubois PM, Freeman 1B. Traumatic abdominal wall hernia. f Trauma 1981;21:72-4

\title{
A case of a yellow patient
}

\section{MMU Chowdhury, S Natarajan}

A 60-year-old woman was referred by her general practitioner with joint pains, muscle aches and her skin becoming yellow on her trunk and limbs over a two-month period. On examination, she had the features shown in her palms (figure 1), elbows (figure 2), and shoulders (figure 3).

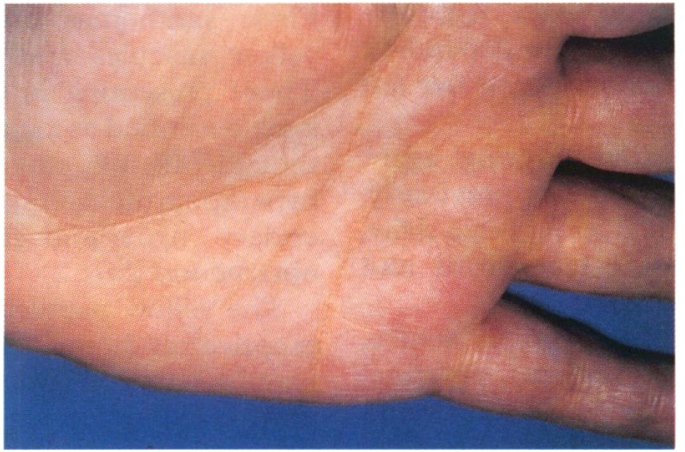

Figure 1

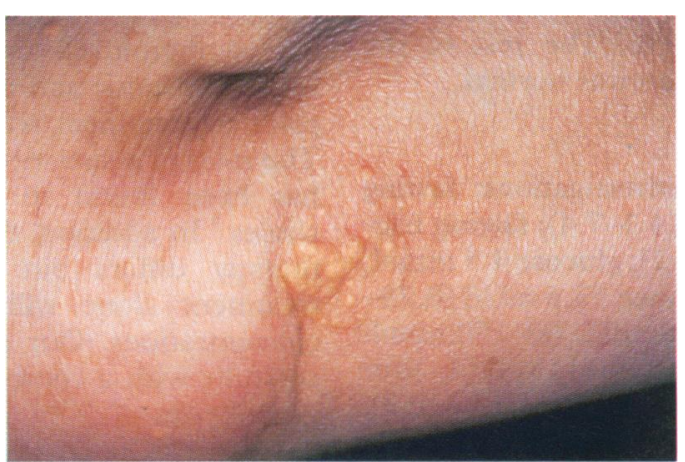

Figure 2

\section{Questions}

1 What are the abnormalities seen in the figures?

2 What further history and examination would be required?

3 What investigations would you perform?

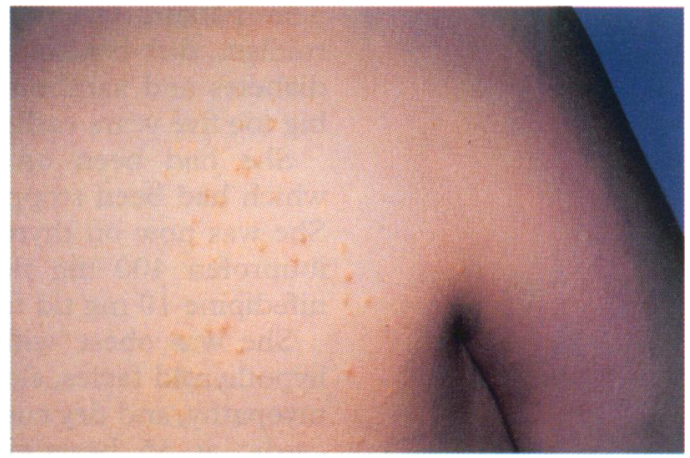

Figure 3 
Answers

QUESTION 1

Figure 1 shows yellow pigmentation along the palmar creases. Figures 2 and 3 show multiple yellow papules on the elbow and shoulder. All of these findings are consistent with the diagnosis of plane (palmar) and eruptive xanthomata.

The various types of xanthomata and their underlying causes are described in box 1 .

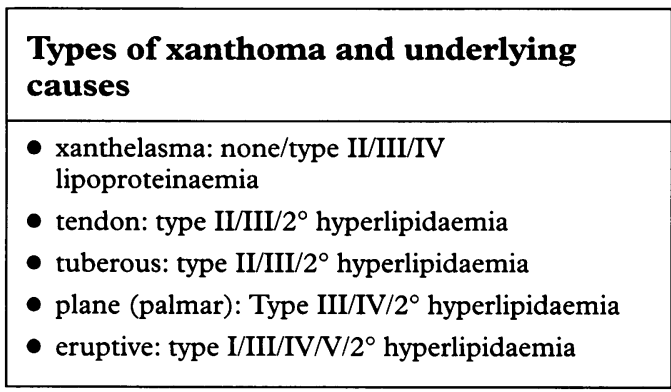

Box 1

QUESTION 2

The patient was known to have hypothyroidism, diet-controlled noninsulin dependent diabetes and xanthoma removal from her left big toe five years earlier.

She had been on a lipid-lowering agent which had been stopped two years previously. She was now on thyroxine $125 \mu \mathrm{g}$ once daily, ibuprofen $400 \mathrm{mg}$ tid for osteoarthritis and nifedipine $10 \mathrm{mg}$ tid for angina.

She was obese with bilateral arcus senilis, hypothyroid facies, slow movements, proximal myopathy, and dry coarse hair. She was bradycardic at 55 beats $/ \mathrm{min}$ and there was slow relaxation of her ankle reflexes. Her blood pressure was $140 / 90 \mathrm{mmHg}$ and there was no evidence of xanthelasma, tendon xanthomata, retinal pathology or goitre. The rest of the cardiovascular examination was normal.

QUESTION 3

She had the following investigations: fasting cholesterol $25.9 \mathrm{mmol} / \mathrm{l}$ (4.3-7.4); fasting triglycerides $8.78 \mathrm{mmol} / 1(0-2)$; glucose $4.5 \mathrm{mmol}$; thyroid-stimulating hormone $53.6 \mathrm{mU} / 1(0.25-$ $2.2 \mathrm{mU} / \mathrm{l})$; thyroxine $54 \mathrm{nmol} / \mathrm{l}(60-150 \mathrm{nmol} /$ 1); $\gamma$-glutamyl transpeptidase $115 \mathrm{IU} /$; liver enzymes, chest X-ray, electrocardiogram, serum electrophoresis, and lipoprotein profile were normal.

\section{Discussion}

Disorders of lipid metabolism commonly present as xanthomas and may be associated with increased risk of arterial vascular disease.

1 Marien KJC, Smeenk G. Plane xanthomata associated with multiple myeloma and hyperlipoproteinaemia. $\mathrm{Br} F \mathrm{Derma}$ tol 1975;93:407-15

2 Polano MK. Xanthomatosis and hyperlipoproteinaemia. Dermatologica 1974;149:1-9.

\section{Secondary causes of hyperlipidaemia}

- diet

- hypothyroidism

- alcohol abuse

- diabetes mellitus

- obesity

- chronic renal disease (eg, nephrotic syndrome)

- chronic liver disease (eg, primary biliary cirrhosis)

- drugs (eg, oral contraceptives, thiazide diuretics, beta blockers, retinoids, cyclosporin, cimetidine)

- multiple myeloma

- pregnancy

- hepatoma

- gout

- Cushing's disease

- acute intermittent porphyria

- anorexia nervosa

- systemic lupus erythematosus

- Gaucher's disease

- glycogen storage disease

Box 2

Plane xanthomas usually appear as orange/ yellow macules and can occur at any site. Those presenting in the palmar creases have been described as xanthoma palmaris and were thought to be pathognomonic of type IV hyperlipoproteinaemia. They also occur in secondary hyperlipidaemias, especially biliary obstruction (box 2). ${ }^{1}$

The yellow papules seen in this patient are eruptive xanthoma. They can present as yellow papules on a red base on any body site, but are most commonly seen on the buttocks, shoulders and extensor aspects of the limbs. These are usually linked with hypertriglyceridaemia and secondary hyperlipidaemias, especially diabetes. $^{2}$

Hypothyroidism is a frequent cause of hyperlipidaemia and this needs to be excluded prior to treatment with lipid-lowering agents. The rates of association of these two conditions has been found to be variable from lipid clinics. ${ }^{3}$ In addition, the response to thyroxine therapy differs but in most patients this treatment alone will suffice to lower lipid concentrations. Our patient was treated successfully with a combination of thyroxine and bezafibrate.

\section{Final diagnosis}

Palmar and eruptive xanthomata secondary to hypothyroidism.

Keywords: xanthomata; secondary hyperlipidaemia; hypothyroidism

3 O'Kane MJ, Neeley RDG, Trimble ERT, Nicolls DP. The incidence of asymptomatic hypothyroidism in new referrals to a hospital lipid clinic. Ann Clin Biochem 1991;28:50911 . 